\title{
LEVEL CURVES OF FUNCTIONS OF BOUNDED CHARACTERISTIC
}

\author{
KARL F. BARTH ${ }^{1}$ AND JAMES G. CLUNIE ${ }^{2}$
}

\begin{abstract}
The authors construct a meromorphic function of bounded characteristic whose level set for 1 contains a sequence of closed Jordan curves which surround the origin and which converge to all of the unit circle. Some sufficient conditions are given for each level set of a meromorphic function of bounded characteristic to end at points.
\end{abstract}

1. Introduction. Suppose $f$ is defined in $D \equiv\{|z|<1\}$. For each $\lambda>0$ we define the level set $L(\lambda, f)=L(\lambda)$ to be $\{z:|f(z)|=\lambda\}$. A component of $L(\lambda)$ is called a level curve. We say that $L(\lambda)$ ends at points of the unit circle $C$ if the maximum diameter of the components of the set

$$
L(\lambda) \cap\{z: 1-\varepsilon<|z|<1\}
$$

tends to 0 as $\varepsilon \rightarrow 0$. A function $f$, holomorphic (meromorphic) and nonconstant in $D$, is said to be in the MacLane Class $\mathcal{L}\left(\mathcal{L}_{m}\right)$ if $L(\lambda)$ ends at points of $C$ for each $\lambda>0$. (See [4, pp. 7-10] and [1, pp. 322-324] for details.)

Let $\Gamma: z=z(t), 0 \leqslant t<1$, be a curve contained in $D$. We say that $\Gamma$ is an asymptotic path if for each $\varepsilon>0$ there exists a $t_{\varepsilon}$ such that $\left\{z(t): t_{\varepsilon}<t<1\right\} \subset\{1$ $-\varepsilon<|z|<1\}$. If $\Gamma$ is an asymptotic path and $\lim _{t \rightarrow 1} f(z(t))=a$ (possibly $\infty$ ), we say that $a$ is an asymptotic value of $f($ on $\Gamma$ ). We say that $f$ has the asymptotic value $a$ at $\zeta,|\zeta|=1$, if there exists an asymptotic path $\Gamma$, ending at $\zeta$, on which $f$ has the asymptotic value $a$. If $f$ is holomorphic (meromorphic) and nonconstant in $D$, we say that $f$ is in the MacLane Class $Q\left(\mathbb{Q}_{m}\right)$ if there exists a dense subset of $C$ at which $f$ has asymptotic values. MacLane proved that $\mathbb{Q}=\mathcal{E}[4$, Theorem 1]. The first author [1, Theorem 3] proved that $\mathbb{Q}_{m} \not \subset \mathcal{L}_{m}$ and $\mathcal{L}_{m} \not \subset \mathbb{Q}_{m}$. Finally, we say that $f \in \mathbb{Q}_{m}^{\prime}\left(\mathcal{L}_{m}^{\prime}\right)$ if $f \in \mathbb{Q}_{m}\left(\mathfrak{L}_{m}\right)$ and $N(r, \infty, f)=0(1)$. Here $N(r, \infty, f)$ denotes the Nevanlinna counting function of the poles of $f$. The first author also proved that $\mathcal{E}_{m}^{\prime} \subset \mathbb{Q}_{m}^{\prime}\left[1\right.$, Theorem 9], but the question whether $\mathbb{Q}_{m}^{\prime} \subset \mathcal{E}_{m}^{\prime}$ remained open. In this paper we shall answer this question negatively by constructing a function of bounded characteristic that is not in $\mathcal{E}_{m}^{\prime}$ (Theorem 5) and shall also derive

Received by the editors November 6, 1979 and, in revised form, June 16, 1980; presented to the Society, October 27, 1978.

1980 Mathematics Subject Classification. Primary 30D50, 30D40.

Key words and phrases. Bounded characteristics, level set, level curve, MacLane Class.

1 The first author gratefully acknowledges support from the National Science Foundation, Grant MCS78-01103.

${ }^{2}$ The authors wish to thank the referee for his many exceedingly helpful suggestions and comments. 
sufficient conditions for a function of bounded characteristic to be in $\ell_{m}^{\prime}$ (Theorems 1,2 and 3). We then apply these results to obtain a uniqueness theorem for functions bounded and holomorphic in $D$ (Theorem 4).

2. Positive results. Let $\left\{\gamma_{n}\right\}$ be a sequence of arcs contained in $D$ and let $\gamma$ be a subarc of $C, \gamma \equiv\left\{e^{i \theta}: \alpha<\theta<\beta\right\}$.

DEFINITION 1. The sequence $\left\{\gamma_{n}\right\}$ converges to $\gamma\left(\gamma_{n} \rightarrow \gamma\right)$ if for each $\varepsilon>0$ there exists an $N(\varepsilon)$ such that

$$
\left.\begin{array}{c}
\gamma_{n} \subset\{1-\varepsilon<|z|<1\} \\
\left|\inf _{\gamma_{n}} \arg z-\alpha\right|<\varepsilon, \quad\left|\sup _{\gamma_{n}} \arg z-\beta\right|<\varepsilon
\end{array}\right\} n>N(\varepsilon) .
$$

Definition 2. We say that $\left\{\gamma_{n}\right\}$ is a Koebe sequence of arcs if it converges to a nondegenerate subarc of $C$.

DEFINITION 3. Let $\gamma$ be a nondegenerate subarc of $C$. We say that $f$, meromorphic in $D$, is in $\mathcal{L}_{m}\left(\mathcal{L}_{m}^{\prime}\right)$ near $\gamma$ if there exists a simply connected open subset $U$ of $D$ with $\bar{U} \cap C=\gamma$ and $f \circ \phi \in \mathcal{L}_{m}\left(\mathcal{L}_{m}^{\prime}\right)$ where $\phi$ is a function that maps $D$ one-to-one and conformally onto $U$.

Now we shall prove the positive results.

TheOREM 1. Suppose $f$ is meromorphic and of bounded characteristic in D. If the interior of some nondegenerate subarc $\gamma$ of $C$ contains no limit points of the poles of $f$, then either $f \in \mathcal{E}_{m}^{\prime}$ near $\gamma$ or $f$ is constant. The conclusion is also valid under the hypothesis that the interior of $\gamma$ contains no limit points of the zeros of $f$.

Proof. If $f$ is nonconstant and $\gamma^{0}$ contains no limit points of the poles of $f$, it follows from a result of MacLane [4, Theorem 1] that $f \in \mathcal{E}_{m}^{\prime}$ near $\gamma$. If $\gamma^{0}$ contains no limit points of the zeros of $f$, it follows from the above that $1 / f \in \mathcal{E}_{m}^{\prime}$ near $\gamma$ and hence that $f \in \mathcal{L}_{m}^{\prime}$ near $\gamma$.

THEOREM 2. Suppose $f$ is of bounded characteristic in $D$. If some neighborhood $V$ (in $D$ ) of some nondegenerate subarc $\gamma$ of $C$ contains no zeros of $f^{\prime}$ and only simple poles of $f$, then either $f \in \mathcal{L}_{m}^{\prime}$ near $\gamma$ or $f$ is constant.

Proof. We can find a simply connected open subset $U$ of $D$ such that $f^{\prime} \neq 0$ in $U, f$ has only simple poles in $U$, and $\bar{U} \cap C=\gamma$. Now we set $F=f \circ \phi$ where $\phi$ maps $\{|Z|<1\}$ one-to-one conformally onto $U$, and we let $\Gamma$ be the preimage of $\gamma$.

Suppose $F \notin \mathcal{L}_{m}^{\prime}$ and $F$ is nonconstant. Then we can find a sequence of simple arcs $\Gamma_{n}$ in $D$ such that $\left\{\Gamma_{n}\right\}$ converges to a subarc of $\Gamma$ and $|F|=\lambda>0$ on $\Gamma_{n}$. To keep the notation simple, we will denote the above subarc of $\Gamma$ also by $\Gamma$ and we suppose that $\lambda=1$. Either some interior point $\zeta_{0}$ of $\Gamma$ is a limit point of zeros of $F$, or Theorem 1 yields the desired conclusion. In the first case, we choose a subsequence of the $\Gamma_{n}$ in such a way that at least one zero lies (in the obvious sense) between $\Gamma_{n}$ and $\Gamma_{n+1}$. Pick a sequence $\left\{a_{n}\right\}$ of zeros of $F$, converging to $\zeta_{0}$ such that $a_{n}$ lies between $\Gamma_{n}$ and $\Gamma_{n+1}$ and let $Q_{n}$ be the point over zero on the Riemann surface $\mathcal{S}$ of $F^{-1}$ corresponding to $a_{n}$. Following MacLane [5, p. 281282], we consider the maximal schlicht disk $\mathscr{D}_{n}$ which can be imbedded in $\mathcal{S}$ with 
center $Q_{n}$. Suppose first that $\mathscr{D}_{n}$ has infinite radius. This would mean that $\delta$ is either the finite plane or the closed Riemann sphere, both of which are impossible. Hence the radius $\rho_{n}$ of $\mathscr{D}_{n}$ must be finite. Since $\mathcal{S}$ has no (algebraic) branch points, it follows that there must be a boundary point $B_{n}$ of $\mathcal{S}$ over $\left\{|w|=\rho_{n}\right\}$. The preimage of the ray from $Q_{n}$ to $B_{n}$ must be an asymptotic path $\Delta_{n}$ beginning at $a_{n}$ on which $F$ has the asymptotic value $B_{n}$. Note that $\Delta_{n}$ can cross at most one of the $\Gamma_{k}$ since the ray from $Q_{n}$ to $B_{n}$ crosses the circle $\{|w|=1\}$ at most once. This means that $\Delta_{n}$ cannot end at an interior point of $\Gamma$. Hence there exist a subsequence $\left\{\Delta_{n_{k}}\right\}$ of $\left\{\Delta_{n}\right\}$ and a sequence of simple arcs $\left\{\tilde{\Delta}_{n_{k}}\right\}$ such that $\tilde{\Delta}_{n_{k}} \subset \Delta_{n_{k}}$ and $\left\{\tilde{\Delta}_{n_{k}}\right\}$ converges to a nondegenerate subarc $\tilde{\Gamma}$ of $\Gamma$. For each $k$, denote the asymptotic value on $\Delta_{n_{k}}$ by $B_{n_{k}}$. There exists a subsequence of the $\left\{B_{n_{k}}\right\}$, which we again denote by $\left\{B_{n_{k}}\right\}$, such that $\left\{\arg B_{n_{k}}\right\}$ tends to a limit, say $\eta$. Thus all the radial limits of $F$ on $\hat{\Gamma}$ must be equal to $e^{i \eta}$, which means that $F$ is constant and the proof is complete.

THEOREM 3. Let $f$ be of bounded characteristic in $D$ and let $R$ be the subset of $C$ at which $f$ has radial limits. If $R$ is residual, then either $f \in \mathcal{L}_{m}^{\prime}$ or $f$ is constant.

REMARK. A subset $F$ of $C$ is said to be of second category evenly if $F \cap \Gamma$ is of second category (in $\gamma$ ) where $\gamma$ is any subarc of $C$. Theorem 3 is true with the formally weaker hypothesis " $R$ is of second category evenly" rather than " $R$ is residual." However, the set where a continuous function in $D$ has radial limits is of

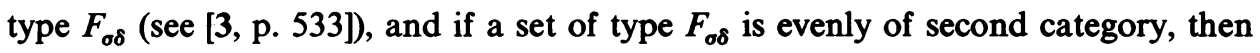
it is also residual.

Proof. Suppose $f \notin \mathcal{L}_{m}^{\prime}$. Then, as before, there exists a sequence of simple arcs $\gamma_{n}$ such $\gamma_{n} \subset D, n \geqslant 1, \gamma_{n} \rightarrow \gamma$ where $\gamma$ is a nondegenerate subarc of $C$ and $|f|=1$ on $\gamma_{n}$. We may again suppose that $\lambda=1$. Note that we must have that $\left|f\left(e^{i \theta}\right)\right|=1$ for all $e^{i \theta}$ in $\gamma$ at which $f$ has a radial limit. Hence, by a Lemma of Collingwood [2, pp. 170-171] there is a subarc $A$ of $\gamma$ such that $f(z)$ is uniformly bounded in a relative neighborhood of $A$ in $D$. Hence by $[4$, p. 10 , Lemma 1$], f \in \mathcal{L}_{m}^{\prime}$ near $A$, a contradiction.

We now apply the above to obtain a uniqueness theorem for functions bounded and holomorphic in $D$.

TheOREM 4. Suppose $f$ and $g$ are bounded and holomorphic in $D$ and suppose there exists a sequence $\left\{\gamma_{n}\right\}$ of Koebe arcs such that $|f|=|g|$ on $\gamma_{n}$. If $f / g$ satisfies the hypotheses of Theorems 1,2 or 3 , then $f=e^{i \theta} g, 0<\theta<2 \pi$.

Proof. Theorems 1,2 or 3 imply that $f / g$ is constant since $f / g \notin \mathcal{L}_{m}^{\prime}$.

\section{Counterexample.}

THEOREM 5. There exists a quotient $f$ of two Blaschke products such that the level set $L(1, f)$ contains infinitely many loops about the origin.

Theorem 5 is a consequence of the following:

TheOREM 6. Let $\eta>0, \varepsilon(0<\varepsilon<1), \delta_{1}$ and $\delta_{2}\left(0<\delta_{1}<\delta_{2}<1\right)$ be given. There exists a function $\phi(z)$ which is a finite quotient of Blaschke factors having the 
following properties:

(i) $\Sigma_{\zeta}(1-|\zeta|)<\eta$, where the sum is taken over the zeros and poles of $\phi(z)$ in $D$;

(ii) $\phi(0)=1$;

(iii) $1-\varepsilon<|\phi(z)|<1+\varepsilon\left(|z|<\delta_{1}\right)$;

(iv) there is an unbranched loop of the level set $\{|\phi(z)|=1\}$ that lies in a channel in $\left\{\delta_{2}<|z|<1\right\}$ whose boundary consists of two loops, one being part of the level set $L\left(1-\varepsilon^{\prime}, \phi\right)$ and the other being part of the level set $L\left(1+\varepsilon^{\prime}, \phi\right)$, where $\varepsilon^{\prime}>0$ and depends only on $\eta$. Furthermore, the channel contains neither zeros nor poles of $\phi$, and it surrounds the origin.

First we show that Theorem 5 is a consequence of Theorem 6 and then prove Theorem 6.

Proof of Theorem 5. Assume that we have a product $\pi_{n}(z)=\phi_{1}(z) \cdots \phi_{n}(z)$ of functions from Theorem 6, where $\eta=\eta_{k}=1 / k^{2}$ for $\phi_{k}(z)$. Assume also that the level set $L\left(1, \pi_{n}\right)$ contains $n$ loops about the origin, each lying in a channel, as in (iv), whose boundary loops lie in $\left\{\left|\pi_{n}(z)\right|<1-\varepsilon_{k}^{(n)}\right\}$ and $\left\{\left|\pi_{n}(z)\right|>1+\varepsilon_{k}^{(n)}\right\}$ for $k=1,2, \ldots, n$.

Now, using Theorem 6, choose as $\phi_{n+1}(z)$ a function $\phi(z)$ with $\eta=1 /(n+1)^{2}$ and $\varepsilon=\min \left\{\varepsilon_{k}^{(n)} / 2^{n}: 1<k<n\right\}$. Also, choose $\delta_{1}$ near enough to 1 to ensure that all the above channels lie in $\left\{|z|<\delta_{1}\right\}$, and choose $\delta_{2}$ so that $\left|\pi_{n}(z)\right|$ is near enough to 1 in $\left\{\delta_{2}<|z|<1\right\}$ to ensure that if $\pi_{n+1}(z) \equiv \pi_{n}(z) \phi_{n+1}(z)$, then the boundary loops of the channel of $\pi_{n+1}(z)$ corresponding to the channel of $\phi_{n+1}(z)$ lie in the sets $\left\{\left|\pi_{n+1}(z)\right|<1-\varepsilon^{\prime} / 2\right\}$ and $\left\{\left|\pi_{n+1}(z)\right|>1+\varepsilon^{\prime} / 2\right\}$. Then $\pi_{n+1}(z)$ is similar to $\pi_{n}(z)$, except for having an additional channel; we now denote the numbers associated with the $n+1$ channels by $\varepsilon_{k}^{(n+1)}(k=1,2, \ldots, n+1)$. Simple considerations show that the function $F(z) \equiv \lim _{n \rightarrow \infty} \pi_{n}(z)$ has all the properties asserted in the theorem.

Proof of Theorem 6. Corresponding to a small positive $\varepsilon$ and a number $c$ in $\left(\frac{1}{2}, 1\right)$, we consider the function

$$
f(z)=\left(\frac{1-z^{2} e^{e}}{1-z^{2} e^{-e}}\right)^{2}\left(\frac{1-z^{2} e^{-(1-c) e}}{1-z^{2} e^{(1-c) e}}\right)^{2}\left(\frac{1-z^{2} e^{-2 c e}}{1-z^{2} e^{2 c e}}\right)
$$

Note that $f$ is a quotient of Blaschke factors and that it is real on the real and imaginary axes. Let $P=e^{-c e}, Q=e^{-c / 2}, R=e^{-(1-c) e / 2}, P^{\prime}=-P, Q^{\prime}=-Q$ and $R^{\prime}=-R$, and note that $P, P^{\prime}, R$ and $R^{\prime}$ are poles of $f$ and $Q$ and $Q^{\prime}$ are zeros of $f$.

LEMMA 1. If $f(x) \leqslant 1$ for small real $x$, then $f^{\prime \prime}(0) \neq 0$ and the level set $L(1, f)$ in $\bar{D}$ is as pictured in Figure 1.

Proof of Lemma 1. The level set $L(1, f)$ is symmetric in each coordinate axis. Also, by the maximum modulus principle, each domain bounded by curves of the level set $L(1, f)$ must contain a zero or a pole. If such a domain contains only zeros or only poles, then $|f(z)|<1$ or $|f(z)|>1$ in the domain. Symmetry excludes the possibility that the set $L(1, f)$ meets the imaginary axis except at 0 . 


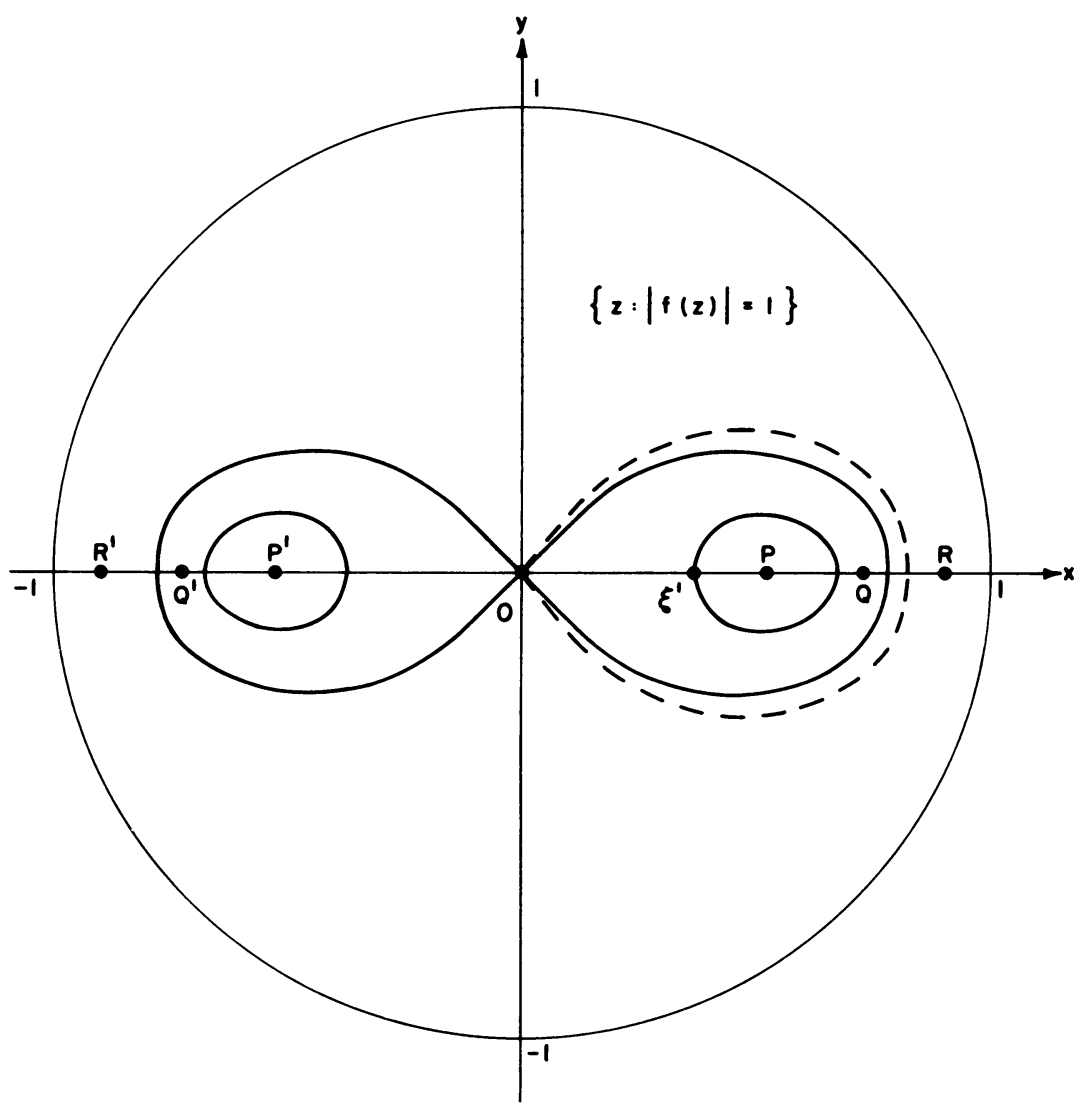

Figure 1

Assume now that $f^{\prime \prime}(0) \neq 0$, so that arcs of the level set $L(1)$ leave 0 in the directions $\theta=\pi / 4,3 \pi / 4,5 \pi / 4$ and $7 \pi / 4$. The arc that leaves the origin in the direction $\pi / 4$ must cross the real axis between $P$ and $Q$ or between $Q$ and $R$. Since $f(x)<1$ for small real $x$, the former possibility is excluded and the figure-eight in the diagram must be part of the level set.

If $f^{\prime \prime}(0)$ were 0 , two arcs of the level set would leave from the origin in directions between 0 and $\pi / 2$. The argument above would apply to each, and there would exist a domain, bounded by the dotted loop and the right loop of the figure-eight as illustrated, that contains neither zeros nor poles. This is impossible. Hence $f^{\prime \prime}(0) \neq$ 0 .

\section{Lemma 2. If $f(x)>1$ for small real $x$, then $f(x)>1$ between 0 and $P$.}

Proof of Lemma 2. Let $L_{0}$ denote the first arc of $L(1)$ that we encounter as we pass around the origin in the positive direction, beginning on the positive real axis and near the origin. As in the proof of Lemma 1, the arc $L_{0}$ loops either only around $P$ or around both $P$ and $Q$. In the latter case, the intersection of $L(1)$ with the interior of $L_{0}$ consists of exactly one simple closed curve $L_{Q}$ that passes around $Q$ but not around $P$. Because $L_{Q}$ is symmetric, it does not meet $O P$. In either case, 
the function $f$ cannot assume the value 1 on the open segment $O P$, and therefore $f(x)>1$ on $O P$.

LEMMA 3. For all small $\varepsilon>0$, the number $c$ can be chosen between $\frac{1}{2}$ and 1 so that $f(x)<1$ for small real $x$ and $f(\xi)=1$ for some $\xi$ in $\left(0, \frac{1}{2}\right]$.

Proof. The series for $f(z)$ at $z=0$ is $1+a_{2} z^{2}+\ldots$, where

$$
\begin{aligned}
a_{2} & =-2\left(e^{\varepsilon}-e^{-\varepsilon}\right)+2\left(e^{(1-c) e}-e^{-(1-c) e}\right)+\left(e^{2 c \varepsilon}-e^{-2 c \varepsilon}\right) \\
& =-4 \sinh \varepsilon+4 \sinh ((1-c) \varepsilon)+2 \sinh 2 c \varepsilon \\
& =-4\left(\varepsilon+\frac{\varepsilon^{3}}{3 !}\right)+4\left[(1-c) \varepsilon+\frac{(1-c)^{3} \varepsilon^{3}}{3 !}\right]+2\left[2 c \varepsilon+\frac{(2 c \varepsilon)^{3}}{3 !}\right]+O\left(\varepsilon^{5}\right)
\end{aligned}
$$

where the bound implied in $O\left(\varepsilon^{5}\right)$; is uniform in $c$ for the range considered, as $\varepsilon \rightarrow 0+$. Algebraic manipulation yields

$$
a_{2}=2 c\left[(c+1 / 2)^{2}-5 / 4\right] \varepsilon^{3}+O\left(\varepsilon^{5}\right) .
$$

Now $\left(c+\frac{1}{2}\right)^{2}-5 / 4$ equals $-11 / 144$ for $c=7 / 12$ and $5 / 16$ for $c=3 / 4$. Hence for all small fixed $\varepsilon>0, a_{2}$ as a function of $c$ is negative for $c=7 / 12$ and positive for $c=3 / 4$.

Corresponding to a small, fixed $\varepsilon>0$, we consider the point $\xi^{\prime}$ of the loop of $L(1, f)$ around $P$ that lies on the segment $O P$, as indicated in Figure 1 . If for $c=7 / 12$ the point $\xi^{\prime}$ lies on $\left[0, \frac{1}{2}\right]$, we take $f$ with this value $c$ to be the function in Lemma 3. If $\xi^{\prime}>\frac{1}{2}$, we consider the value $f\left(\frac{1}{2}\right)$ as a function of $c$ on the interval $7 / 12 \leqslant c \leqslant 3 / 4$. Because $f\left(\frac{1}{2}\right)$ is a continuous function of $c$ and is by virtue of Lemmas 1 and 2 less than 1 at $c=7 / 12$ and greater then 1 at $c=3 / 4$, we can choose $c$ so that $f\left(\frac{1}{2}\right)=1$. The function $f$ with this value of $c$ is the desired function. We now write $\frac{1}{2}=\xi$.

If we were to map $D$ onto itself by a linear fractional transformation that carries $\xi$ to the origin, we would almost have the desired function. The right-hand loop of the figure-eight would be carried into the level curve for $\lambda=1$ surrounding the origin. However, the branching at the double point makes it difficult to cope with approximation problems by placing it in a channel. To overcome this difficulty we modify the construction as follows.

If $\rho(0<\rho<1)$ is near to 1 , the level set $\{|f(z)|=\rho\}$ will contain a loop $L_{1}$ just outside the unit circle $C$, a loop $L_{2}$ just inside the right-hand loop of the figure-eight (see Figure 1) which is part of $\{|f(z)|=1\}$ and a loop $L_{3}$ just outside the loop around $P$ (see Figure 1) which is also part of $\{|f(z)|=1\}$. Let $\xi_{0}$ be the nearer to 0 of the two points of $L_{3}$ on $(0,1)$, so that $\xi_{0}$ is very near to $\xi$ if $\rho$ is very near to 1 . Now let $\Psi(z)$ be a one-to-one conformal map of $D$ onto the inside of $L_{1}$ such that $\Psi(0)=\xi_{0}$, and consider $g=(1 / \rho) f \circ \Psi$.

Since $|g(z)|=1$ on $C$, it follows that $g(z)$ is a quotient of Blaschke factors with the same number of zeros and poles as $f(z)$. Since (i) is satisfied by $f(z)$, it will also be satisfied by $g(z)$ if $\rho$ is chosen near enough to 1 ; since this also ensures that $\xi_{0}$ is not much larger than $\frac{1}{2}$, we may assume that $\xi_{0} \in(0,3 / 4)$. Also, (ii) is satisfied, i.e. $g(0)=1$. Finally, (iii) and (iv) can be satisfied by setting $\phi(z)=g\left(z^{k}\right)$ with $k \in N$ 
sufficiently large. We remark that $\phi$ trivially satisfies (ii), and straightforward calculations show that if $g(z)$ satisfies (i), then so does $g\left(z^{k}\right)$. Thus the proof is complete.

\section{REFERENCES}

1. K. F. Barth, Asymptotic values of meromorphic functions, Michigan Math. J. 13 (1966), 321-340.

2. E. F. Collingwood, On the linear and angular cluster sets of functions meromorphic in the unit circle, Acta Math. 91 (1954), 165-185.

3. F. Herzog and G. Piranian, Sets of radial continuity of analytic functions, Pacific J. Math. 4 (1954), 533-538.

4. G. R. MacLane, Asymptotic values of holomorphic functions, Rice Univ. Studies 49 (1963), 83 pp.

5. Exceptional values of $f^{(n)}(z)$, asymptotic values of $f(z)$, and linearly accessible asymptotic values, Mathematical Essays Dedicated to A. J. MacIntyre (H. Shankar, Ed.), Ohio Univ. Press, Athens, Ohio, 1970.

Department of Mathematics, Syracuse University, Syracuse, New York 13210

Department of Mathematics, Imperial College of Science and Technology, london SW7, ENGLAND 